\title{
Simultaneous identification of phenolic and flavonoid contents in bee pollen by HPLC-ESI-MS data.
}

\author{
Shagufta Perveen ${ }^{1 *}$, Raha Orfali ${ }^{1 *}$, Areej Mohammad Al-Taweel ${ }^{1}$, Afsar Khan ${ }^{2}$, Bandar Alghanem ${ }^{3}$, \\ Hayat Shaibah ${ }^{3}$ \\ ${ }^{1}$ Department of Pharmacognosy, College of Pharmacy, King Saud University, Riyadh, Saudi Arabia \\ ${ }^{2}$ Department of Chemistry, COMSATS University Islamabad, Pakistan \\ ${ }^{3}$ Medical Core Facility and Research Platforms, King Abdullah International Medical Research Centre/King Saud Bin \\ Abdulaziz University for Health Sciences, Ministry of National Guard Health Affairs, Riyadh, 11426, Saudi Arabia
}

\begin{abstract}
A HPLC-MASS method has been developed for the characterization of secondary metabolites present in the methanol extract of honey bee pollen pallet. As a result, three new and five known compounds were identified or tentatively characterized by using ESIMS data. The chemical composition of methanol extract of pollen pallet is presented for the first time by using the LC-ESI-MS method. In conclusion, LC-MS/MS based chemical screening of diverse bioactive compounds in matrices of bee pollen pallet methanol extract would appear to be an effective approach for discovering new/known compounds. A high percentage of the detected compounds corresponded to glycosylated flavonoids and phenolic were identified.
\end{abstract}

Keywords: Bee pollen, Flavonoid glycosides, Phenolic glycosides, ESI-MS.

Accepted on June 20, 2019

\section{Introduction}

Complementary and alternative herbal treatments have excellent cures for much disease with required nutrients of human body. Honey and bee wax are the most common products of honey bees used as a medicine food and cosmetics. Pollen pallet, royal jelly, hives, propolis are also demanding apicultural products of honey bee. Bee (Apis mellifera) collects pollen from the stamens of different flowering plants and used it for feeding larvae. The pollens are found in different colors (dark brown to pale yellow) with the 10-200 $\mu \mathrm{m}$ diameters. Bee Pollen is considered as a complete food due to its comprehensive and balanced nutrients. Due to healthy and rich constituents, it is considered as an immune system builder that enhances vitality. Bee Pollen has been traditionally used in many cultures throughout the world. It usually use as raw or with hot/cold drinks and in breakfast with cereals. Hippocrates regarded it as the father of modern medicine and prescribed it for healing. Chinese people also using bee pollen as medicine for thousands of years and known as perfectly complete food. It contains up to $55 \%$ carbohydrate (Fructose, glucose, sucrose), vitamins (C, B1, B2, B3, B6 and E), 50\% of protein, many fatty acids and it traditionally use as medicine for boosting immunity and eliminating allergies. Different biological activities of the bee pollen were established, such as antimicrobial, anti-inflammatory, anti-mutagenic, antihistopathologic, antinociceptive and antioxidant. The responsibility for the biological activities is attributed to phenolic contents in it [1].

A plethora of analytical methods based on thin layer chromatography (TLC) and reversed-phase high-performance liquid chromatography (RPHPLC)/UV - Vis diode array detection (DAD), capillary electrophoresis (CE); nano-liquid chromatography, HPLC-diode array detector (DAD) and HPLC with photodiode-array detection (PDA) coupled to an ion trap mass spectrometry have been developed and applied for separation of bee pollen phenolic compounds [2]. HPLCMS technologies are extremely important for characterization and identification of different food materials because characterization of secondary metabolites is the desirable goal. It is very sensitive and high-throughput method with possibility of identification of the compounds present in food daily used by consumers. To the best of our knowledge, LC-ESI-MS profiling of methanol extract of Bee pollen pallet is not reported in literature, the purpose of the present study was to explore the chemical composition of phenolic constituents present in $80 \%$ hydromethanolic extract of pollen pallet, so that appropriate analytical assays could be developed and applied to the pharmaceutical production and quality control of this botanical product. HPLC-ESI-MS spectrometry was used for the structural elucidation of the compounds. 


\section{Materials and Methods}

We purchased pollen pallets from the local market. Reagents acetonitrile LC-MS grade (purity 99.99\%) was purchased from Thermo Scientific (Rockford, IL, USA). Formic acid was purchased from Sigma-Aldrich (St. Louis, MO, USA). Aqueousmethanol (3:7 ratios) for extraction purpose was prepared by mixing methanol $(70 \mathrm{ml})$ with Millipore water $(30$ $\mathrm{ml})$.

\section{Preparation of aqueous - methanol extract of pollen}

Pollen pallets were weighed to $100 \mathrm{~g}$ and washed with water to remove the dirt and then dried with filter paper. Clean pallets were kept for two hours at room temperature for drying it completely. Then pallets were ground into powder with precooled mortar and pestle. Obtained fine powder was used as the raw material for extraction with $500 \mathrm{ml}$ of methanol $(70 \%)$ and millipore water $(30 \%)$ as a solvent. The mixtures of solvent and pallets were vortexed and kept in cold water bath for $20 \mathrm{~min}$ followed by the cell breaking treatments. The phenolic constituents were extracted by ultrasound-assisted extraction. Ultrasonication used for breaking of cells and it proceeded on an ice bath for $20 \mathrm{~min}$. After completion of sonication process, the mixtures were kept for centrifugation for $10 \mathrm{~min}$ to obtain clear supernatants. This extraction process was repeated thrice to ensure that complete secondary metabolites were extracted. On completion of centrifugation, clear supernatant was collected and kept in $-80^{\circ} \mathrm{C}$ for freezing. Finally, freeze samples were kept for 20 hours at freeze dryer for lyophilization to yield the dry powder $(300 \mathrm{mg})$. The resulting extracts were stored at $-20^{\circ} \mathrm{C}$ until further analysis.

\section{Results and Discussion}

\section{Identification of compounds}

Plantbased metabolomics study is very challenging as compared with human-based metabolomics because plant extracts contain a wide range of secondary metabolites including terpenes, flavonoids, fatty acids, carbohydrates, amino acids and phenolic. Identification of each constituent in plant metabolomics is a cumbersome process due to the lack of a webbased database. However, there are many published literature that can guide us to assign and identify the secondary metabolites in different part of plant material. In our current study, HPLC-Mass experiments were performed to assign the chemical constituents in aqueousmethanol extract of honey bee pollen pallets. Molecular ions signal in negative mode, retention time (tR) and fragment ion masses of identified compounds were shown for the methanol extract of pollen pallets granules (Table 1). A new phenolic glycoside, 3,4,5trihydroxycinnamic acid diglycoside (1), two new flavonoid derivatives (6) and mono-acetylated kaempferol diglycoside (8), while five known compounds namely; 3-hydroxycinnamic acid dirhamnoside (2), ellagic acid diglycoside (3), ellagic acid monoglycoside (4), taxifolin diglycoside (5), isorhamnetin diglycoside (7) were identified by HPLC-ESI-MS (Figure 1). The compounds were verified by online database such as dictionary of natural product, Sci finder, scopus etc.

Table 1. Mass data for characterization of compounds in bee pollen by HPLC-ESI-MS.

\begin{tabular}{|c|c|c|c|c|c|}
\hline \# & $\begin{array}{l}\text { Negative molecular ion [M-H]- } \\
\qquad \mathrm{m} / \mathrm{z}\end{array}$ & Fragment ion $\mathrm{m} / \mathrm{z}$ & Name of compounds & Compound & $\operatorname{tR}(\mathrm{min})$ \\
\hline 1 & 489.18 & $537,358,341,195$ & 3,4,5-Trihydroxycinnamic acid diglycoside & New & 6.35 \\
\hline 2 & 455.17 & 523,409 & 3-Hydroxycinnamic dirhamnoside acid & Known & 8.85 \\
\hline 3 & 625.16 & 609,593 & Ellagic acid diglycoside & Known & 19.8 \\
\hline 4 & 463.09 & & Ellagic acid monoglycoside & Known & 20.13 \\
\hline 5 & 598.26 & 566,478 & Taxifolin diglycoside & Known & 22.52 \\
\hline 6 & 582.26 & 462 & Flavonoid derivative & New & 23.13 \\
\hline 7 & 593.27 & $661,494,315$ & Isorhamnetin diglycoside & Known & 25.8 \\
\hline 8 & 636.31 & 593,431 & Mono-acetylated kaempferol diglycoside & New & 27.53 \\
\hline
\end{tabular}




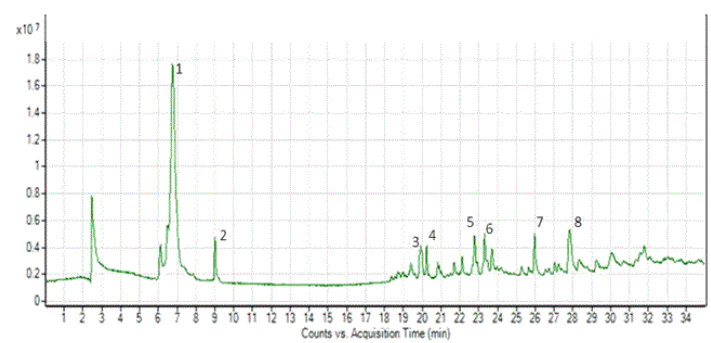

Figure 1. HPLC chromatogram of the hydro methanolic extract of honey bee pollen pallet.

HPLC-ESI-MS analysis of signal 1 revealed the presence of negative molecular ion $\mathrm{M}-\mathrm{H}-$ at $\mathrm{m} / \mathrm{z} 489\left(\mathrm{C}_{20} \mathrm{H}_{25} \mathrm{O}_{14}\right)$ and negative fragment ions at $\mathrm{m} / \mathrm{z}$ of 358,341 and 195 , corresponding to trihydroxycinnamic acid diglycoside with retention time $6.3 \mathrm{~min}$. The fragment ion at $\mathrm{m} / \mathrm{z} 341$ $\left(\mathrm{C}_{15} \mathrm{H}_{17} \mathrm{O}_{9}\right)$ appeared due to the loss of one glycoside which can be arabinose or xylose, while the signal at $\mathrm{m} / z \quad 195$ $\left(\mathrm{C}_{9} \mathrm{H}_{7} \mathrm{O}_{5}\right)$ appeared after the loss of both sugar moieties. It further showed the negative ion signal at $\mathrm{m} / \mathrm{z} \quad 537$ corresponding to $\mathrm{M}-\mathrm{H}+2 \mathrm{Na}$. This compound was identified as a new phenolic constituent; 3,4,5-trihydroxycinnamic acid diglycoside (1) (Figure 2) and, to our knowledge, is here reported for the first time. The mass spectrum of the signal 1 is shown in Figure 1 in negative ion.

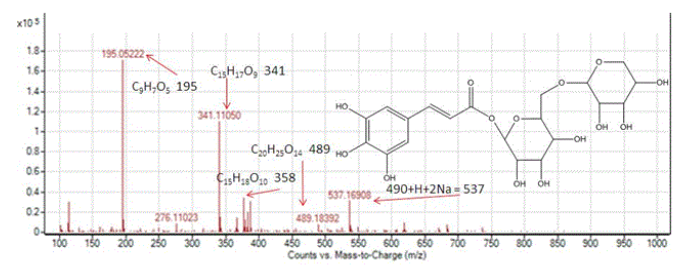

Figure 2. The mass spectrum of the signal 1.

The peak eluting at 8.8 min displayed a negative molecular ion at $\mathrm{m} / \mathrm{z}$ of 455 , which corresponds to hydroxycinnamic acid bound to a disaccharide composed of two hexose unit (rhamnoside), and a fragment ion at $\mathrm{m} / \mathrm{z}$ of 409 corresponds to the loss of three hydroxyl moieties. It further showed the negative ion signal at $m / z 523$ corresponding to $\mathrm{M}+\mathrm{H}+3 \mathrm{Na}$. This compound was identified as a hydroxycinnamic acid dirhamnoside (2) (Figure 3), which has not previously identified and we reported here for the first time. The peak eluting at a retention time of 19.8 min was identified as, ellagic acid diglycoside (3) since it displayed a negative molecular ion at $\mathrm{m} / \mathrm{z}$ of $625\left(\mathrm{C}_{26} \mathrm{H}_{25} \mathrm{O}_{18}\right)$, which corresponds to ellagic acid bound to a disaccharide moiety consist on two hexose units. Two fragment ion peaks appeared at m/z 609 (after the loss of one hydroxyl group) and $\mathrm{m} / \mathrm{z} 593$ (after the loss of two hydroxyl group) (Figure 4). This compound has previously been identified in the fruits of Moroccon Pistacia atlantica with same retention time at $19.7 \mathrm{~min}$ [3].

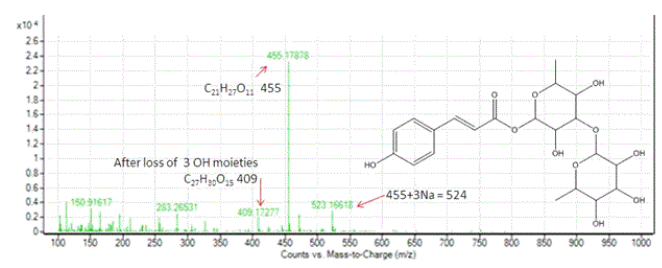

Figure 3. The mass spectrum of the signal 2.

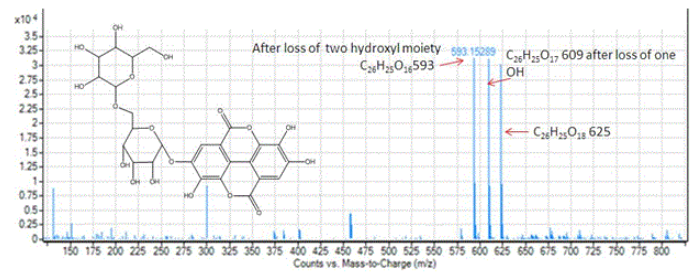

Figure 4. The mass spectrum of the signal 3.

Compound 4 have molecular ion $[\mathrm{M}-\mathrm{H}]-$ at $\mathrm{m} / \mathrm{z} 463$ and characterized as ellagic acid monoglycoside (Figure 5) and it has isolated before from P. atlantica [3].

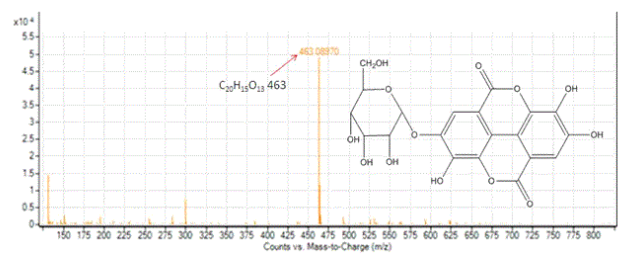

Figure 5. The mass spectrum of the signal 4.

Compound 5 displayed a molecular ion at $\mathrm{m} / \mathrm{z} 598$ $\left(\mathrm{C}_{26} \mathrm{H}_{30} \mathrm{O}_{16}\right)$, and suffered the loss of two hydroxy, yielding a signal at $\mathrm{m} / \mathrm{z} 566$ (Figure 6). However, the aglycone could not be identified and it tentatively characterized as taxifolin diglycoside. This is a polyphenolic compound which commonly found in foods of plant origin and in pines of Pinus sylvestris [4]

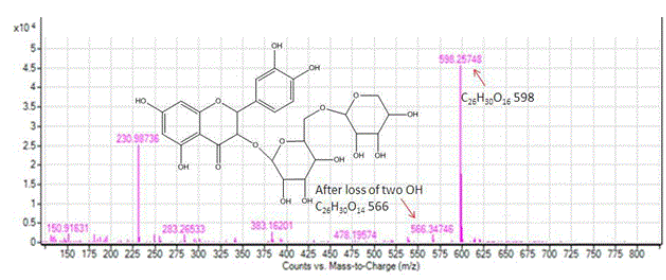

Figure 6. The mass spectrum of the signal 5.

Compound 6 was characterized as flavonoid with monobenzoylated pyranose sugar due to the presence of molecular ion at $m / z 582\left(\mathrm{C}_{29} \mathrm{H}_{26} \mathrm{O}_{13}\right)$ and fragment ions at $m / z$ 462 (after loss of benzoyl group) (Figure 7). This compound was identified as a new flavonoid based natural product and, to our knowledge, is here reported for the first time. 


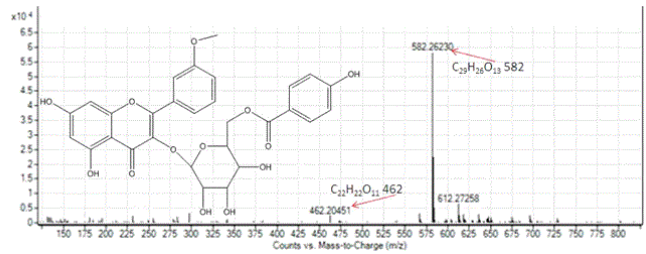

Figure 7. The mass spectrum of the signal 6.

Compound 7 displayed the deprotonated molecular ion at $\mathrm{m} / \mathrm{z}$ 593 and exhibited fragment ion at $m / z 315$. This fragmentation pattern is consistent with a flavonoid; isorhamnetin diglycoside $\left(\mathrm{C}_{27} \mathrm{H}_{29} \mathrm{O}_{15}\right)$ (Figure 8). After a loss of both hexoside signal 7 yield a fragment ion at $\mathrm{m} / \mathrm{z} 315$, which is similar to isorhamnetin $\left(\mathrm{C}_{16} \mathrm{H}_{12} \mathrm{O}_{7} \mathrm{~m} / z\right.$ 316). Taking into account, this compound has an isorhamnetin aglycone part with internal sugar rhamnose and the terminal sugar could be arabinose or xylose [5].

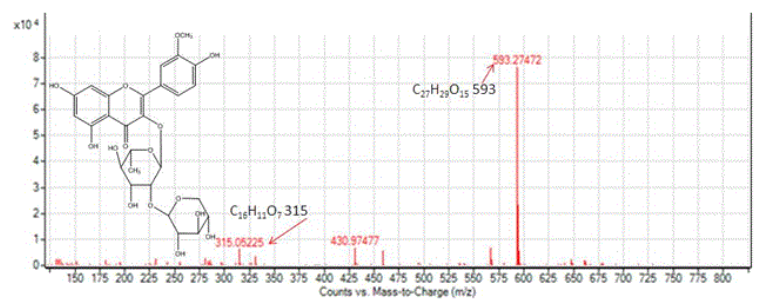

Figure 8. The mass spectrum of the signal 7.

Compound 8 was characterized as kaempferol 3-O-(mono acetyl glucosyl-O-rhamnopyranoside $\left(\mathrm{C}_{29} \mathrm{H}_{32} \mathrm{O}_{16} \mathrm{~m} / \mathrm{z}\right.$ 636). According to mass data, with an $[\mathrm{M}-\mathrm{H}]-$ ion at $\mathrm{m} / \mathrm{z} 593$ (due to the loss of acetyl group) and main fragment ion at $\mathrm{m} / \mathrm{z} 431$ (due to the loss of glucose and acetyl moieties), it confirmed that compound 8 is consisting on mono- acetylated kaempferol diglycoside (Figure 9). The same compounds with different connectivity of sugar unit have been previously identified in the genus Prunus by using UPLC-DAD-QTOF/MS [6].

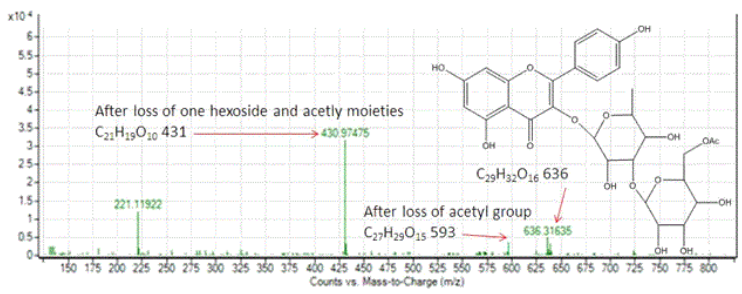

Figure 9. The mass spectrum of the signal 8.

A large number of research publications about the bee pollen analysis during last two decades demonstrate rediscovery of the bee pollen. Analysis of the chemical ingredients in different extracts is a challenging task, because of the diversity of the secondary metabolites and seasonal variation of the chemical content. The identifications of chemical constituents in pollen pallet of honey bee have been recently published [7,8]; however, as previously stated the phenolic and flavonoid profile of these pallets has not been extensively investigated by HPLC-ESI-MS experiments. Many phenolic acids; gallic, chlorogenic, caffeic, ferulic, syringic, synapic, 3,4dihydrobenzoic, and 2-hydroxycinnamic acid and flavonoids; rutin, naringenin and quercetin were identified in the bee pollen produced in Latvia, Spain, Lithuania and China by using HPLC-ECD electrochemical detection and chemometric analysis [2]. In Zea mays monofloral bee pollen from Egypt, 3,4-dimethoxycinnamic acid was the predominant phenolic component $(45.8 \mathrm{mg} / \mathrm{mL})$ in the pollen extract [7]. It is also worth noting that, in our study, a noticeable number of phenolic and flavonoid glycosides were identified in the pallets. Overall findings indicate that pollen pallets can be utilized as the basis for ingredients of functional foods or as antioxidant additives. A recently published paper confirms bee pollen pallets as a good complement to diet and characterized its chemical composition and they calculated the percentages of amino acids, fatty acids and minerals $[8,9]$.

\section{LC-QTOF-MS method}

Pollen pallets granules were grinded and dissolved in methanol at a concentration of $5 \mathrm{mg} / \mathrm{mL}$ and filtered through a $0.45 \mu \mathrm{m}$ cellulose acetate filter (Millipore, Billerica, MA) and then subjected to HPLC analysis. Samples were analyzed by Agilent1260 Infinity HPLC chromatographic system (Agilent, Germany) hyphenated to Agilent 6530 Quadrupole Time of Flight (Agilent, Singapore). $5 \mu \mathrm{l}$ of the sample was injected into Agilent Zorbax SB-C18 column $(4.6 \mathrm{~mm} \times 150 \mathrm{~mm}, 1.8$ $\mu \mathrm{m})$ and the temperature was kept at $40^{\circ} \mathrm{C}$. Separation was performed using the following elution gradient; $0-1 \mathrm{~min}, 5 \% \mathrm{~B}$; 1-25 min, 5-100\% B; 25-30 min, 100\%B; 30-31 min, 100-5\%B; $31-35 \mathrm{~min}, 5 \% \mathrm{~B}$ using mobile phase $\mathrm{A}(0.1 \% \mathrm{HCOOH}$ in water) and mobile phase $\mathrm{B}(0.1 \% \mathrm{HCOOH}$ in Acetonitrile) and the flow rate was as $200 \mu \mathrm{l} / \mathrm{min}$. MS1 (full scan) was used as Acquisition mode and the mass was ranged from 100-1000 m/z with scan rate=1 (Spectra/sec). The mass spectrometer parameters were set as following: Gas Temperature $=300^{\circ} \mathrm{C}$; Gas flow $=8$ I/min; Nebulizer $=35$ psig; Sheath Gas Temperature $=350$ and SheathGas flow was11. MS spectra were recorded in negative ion mode.

\section{Conclusion}

It is confirmed that the biological properties of bee pollen is due to the high content of phenolic and flavonoid glycosides; which makes it highly antioxidant food material. HPLC-ESI spectrometry was utilized for the identification and assignment of eight polar secondary metabolites in a snapshot without resorting to chromatographic column separation of these constituents. Identification of 3,4,5-trihydroxycinnamic acid diglycoside is described as phytochemical component of for the first time. These data indicate that bee pollen may have a very important role to play in health beneficial effects. This HPLC-MS method is an excellent tool for fast and rapid identification of phenolic and flavonoid glycosides in honey bee pollen pallet and provides references for the quality evaluation of other natural products. In conclusion, Nuclear 
Magnetic resonance (NMR) studies are recommended to improve assignment of secondary metabolites further in the complex mixture.

\section{Acknowledgments}

This research project was supported by a grant from the Research Center of the Female Scientific and Medical Colleges, Deanship of Scientific Research, King Saud University.

\section{References}

1. Feas X, Vazquez-Tato MP, Estevinho L, Seijas JA, Iglesias A. Organic bee pollen: botanical origin, nutritional value, bioactive compounds, antioxidant activity and microbiological quality. Molecules 2012; 17: 8359-8377.

2. Kaskoniene V, Ruockuviene G,Kaskonas P, Akuneca I, Maruska A. Chemometric analysis of bee pollen based on volatile and phenolic compound compositions and antioxidant properties. Food Anal Methods 2015;8: 1150-1163.

3. Khallouki F,Breuer A, Merieme E, Ulrich CM,Owen RW. Characterization and quantitation of the polyphenolic compounds detected in methanol extracts of Pistacia atlantica Desf. fruits from the Guelmim region of Morocco. J Pharm Biomed Anal 2017; 134: 310-318.

4. Auger MA, Jay-Allemand C, Bastien C, Geri C. Quantitative variations of taxifolin and its glucoside in Pinus sylvestris needles consumed by Diprion pini larvae. Ann For Sci; 51: 135-146.
5. Antunes-Ricardo M, Gutierrez-Uribe JA, Martinez-Vitela C, Serna-Saldivar SO. Topical Anti-Inflammatory Effects of Isorhamnetin Glycosides Isolated from Opuntia ficusindica. Biomed Res Int 2015; 2015: 1-9.

6. Jang GH, Kim HW, Lee MK, Jeong SY, Bak AR, Lee DJ, Kim JB. Characterization and quantification of flavonoid glycosides in the Prunus genus by UPLC-DADQTOF/MS. Saudi J Biol Sci 2018; 25: 1622-1631.

7. Mohdaly AA, Mahmoud AA, Roby MHH, Smetanska I, Ramadan MF. Phenolic extract from propolis and bee pollen: composition, antioxidant and antibacterial activities. J Food Biochem 2015; 39: 538-547.

8. Denisow B, Denisow-Pietrzyk M. Biological and therapeutic properties of bee pollen: a review. J Sci Food Agric 2016; 96: 4303-4309.

9. Yang K, Wu D, Ye X, D. Liu, Chen J, Sun P. Characterization of chemical composition of bee pollen in China. J Agric Food Chem 2013; 61:708-718.

\section{*Correspondence to}

Shagufta Perveen and Raha Orfali

Department of Pharmacognosy

College of Pharmacy

King Saud University

Saudi Arabia 